\title{
Metabolic effects of chronic exercise on adiponectin and cardiovascular risk in obese subjects with impaired glucose tolerance
}

\author{
A. M. McNeilly ${ }^{1}$, C. M. McClean ${ }^{1}$, J. McEneny $^{2}$, T. R. Trinick ${ }^{3}$, E. Duly ${ }^{3}$, M. H. Murphy ${ }^{1}$ \\ and G. W. Davison ${ }^{1}$ \\ ${ }^{1}$ Sport and Exercise Sciences Research Institute, University of Ulster, Jordanstown BT37 OQB, UK, ${ }^{2}$ Nutrition and \\ Metabolism Group, Centre for Public Health, Queen's University, Grosvenor Road, Belfast BT12 6BJ, UK and \\ ${ }^{3}$ Ulster Hospital, Dundonald BT16 1RH, UK
}

Globally the number of individuals with diabetes is expected to double over the next two decades, increasing from 194 million in 2003 to 380 million in 2025 , the majority of whom will have non-insulin-dependent diabetes mellitus ${ }^{(1)}$. Given the relationship between obesity and the development of diabetes, physical activity intervention in this population is essential.

The aim of the present study was to examine the metabolic effects of chronic exercise on adiponectin, body composition and other cardiovascular risk variables including glycaemic control and absolute insulin in obese patients with impaired glucose tolerance (IGT).

Eleven obese subjects (six males, five females) diagnosed with IGT were recruited following ethical approval. Subjects were required to undertake a 12-week exercise training programme involving 30 min moderate-intensity exercise equivalent to $65 \%$ of maximum heart rate $\left(\mathrm{HR}_{\max }\right)$ on $5 \mathrm{~d}$ of the week. Anthropometric measurements and blood pressure (BP) were measured before and following intervention. Venous blood samples were drawn for the determination of glucose, $\mathrm{HbA}_{1 \mathrm{C}}$, blood lipids and insulin. Multimeric adiponectin concentration $(\mathrm{mg} / \mathrm{ml})$ was assessed using a commercially-available ELISA kit (Alpco Diagnostics, Salem, NH, USA) with a Triturus ${ }^{\mathrm{TM}}$ automated ELISA machine (Grifols UK Ltd, Cambridge, UK).

Following intervention body mass, total body fat, systolic and diastolic BP and waist and hip measurements decreased $(P<0.05)$. Plasma concentrations of glucose, TAG, total cholesterol, LDL-cholesterol (mmol/l) and insulin (mU/l) were not significantly different $(P>0.05)$. All multimeric forms of adiponectin (total, high, total:high, medium and low) were not different following 12 weeks of exercise.

\begin{tabular}{lcrrr}
\hline & \multicolumn{2}{c}{ Baseline } & \multicolumn{2}{c}{ Post intervention } \\
\cline { 2 - 3 } Variable & Mean & SD & Mean & 15.5 \\
\hline Body mass (kg) & $89.0^{\mathrm{a}}$ & 16.6 & $86.2^{\mathrm{b}}$ & 6.2 \\
BMI $\left(\mathrm{kg} / \mathrm{m}^{2}\right)$ & $32.4^{\mathrm{a}}$ & 7.1 & $31.0^{\mathrm{b}}$ & 9.5 \\
Total body fat (\%) & $47.9^{\mathrm{a}}$ & 9.9 & $44.0^{\mathrm{b}}$ & 13.5 \\
Waist $(\mathrm{cm})$ & $102^{\mathrm{a}}$ & 16.6 & $96.9^{\mathrm{b}}$ & 13.8 \\
Hip $(\mathrm{cm})$ & $118^{\mathrm{a}}$ & 12.6 & $109^{\mathrm{b}}$ & 14.9 \\
Systolic BP (mmHg) & $145^{\mathrm{a}}$ & 14.5 & $136^{\mathrm{b}}$ & 16.5 \\
Diastolic BP (mmHg) & $94.5^{\mathrm{a}}$ & 14.8 & $87.7^{\mathrm{b}}$ & 0.6 \\
Glucose (mmol/l) & 5.63 & 0.6 & 5.61 & 0.3 \\
HbA 1 (\%) & 5.63 & 0.4 & 5.63 & 10.1 \\
Insulin (mU/l) & 16.5 & 7.5 & 1.27 & 0.24 \\
HDL-cholesterol (mmol/l) & 1.23 & 0.2 & 5.88 & 2.12 \\
Total adiponectin (mg/l) & 4.91 & 2.31 & 2.88 & 1.32 \\
High-molecular-weight (HMW) adiponectin & 2.11 & 1.82 & 0.47 & 0.09 \\
Total:HMW adiponectin & 0.38 & 0.19 &
\end{tabular}

a,b Values in the same row with different superscript letters were significantly different (paired samples $t$ test; $P<0.05$ ).

The findings suggest that 12 weeks of exercise is effective in eliciting positive changes in body composition and haemodynamic properties. Further research is required to determine the metabolic changes associated with prolonged exercise in this population.

1. Institute of Grocery Directors (2006) Food Consumption Survey 2005. Watford, Herts: IGD. 